\title{
A GDP research network examines patients' perceived oral health
}

\author{
A general dental practice research network: impact of oral health in general dental practice patients \\ E. J. Kay, N. Ward and D. Locker Br Dent J 2003; 194: 621-625
}

\section{Objective}

To measure the subjective impact of oral health in a group of patients attending general dental practices in the North West of England and to investigate the attributes of dentists and practices in order to examine how such attributes might relate to patients' subjective perceptions of oral health.

\section{Design}

Fifteen general dental practices conducting a simultaneous survey of attending patients and 15 practitioners from these practices providing information about their attitudes to treatment, prevention and various aspects of their surgery.

\section{Setting}

General dental practice.

\section{Outcome measures}

Patient subjective impact scores. Relationships between practice and practitioner variables and patients' subjectively perceived oral health.

\section{Results}

Fifteen practitioners with diverse practice attributes provided data on 718 patients. The mean total oral health impact score was 18.4. Twenty two per cent of patients had experienced pain in the four weeks before the survey and 11\% had been unable to chew some foods. Fifty five per cent of the surveyed population had, in the previous year, worried about the appearance of their mouth and $65 \%$ had worried about their oral health in general. Dentists' beliefs were related to patient impact scores but practice attributes were not significantly associated with patients' impacts.

\section{Conclusions}

Fourteen percent of the differences in patients' subjectively perceived oral health can be attributed to dentist attitudes and attributes. Further research regarding the influence of dentists' personality and professional beliefs on patients well-being needs to be undertaken.

\section{IN BRIEF}

- General dental practitioner research networks are capable of producing and analysing large and complex datasets.

- Patients' subjectively perceived oral health is affected by the attributes and attitudes of their dentist.

- Longitudinal studies of patients receiving treatment from GDPs would provide important data about the effectiveness of dental care.

\section{COMMENT}

This paper is very interesting for two very important reasons; firstly, the findings of the study, which were patient centred, will perhaps, begin to inform the future provision of dental services. Secondly, the study design and data collection used a general dental practitioner (GDP) research network, which adds considerable value to the data and was a valuable training exercise for the practitioners involved.

The study was based around a patient questionnaire for which there was an extremely high response rate $(95.7 \%)$, as the authors point out, this is probably due to the effectiveness of motivated practitioners brought together in a research network. The high response rate gives confidence in the findings of the study.

Surprisingly the patients' subjective perceptions of their oral health revealed that $65 \%$ worried about their level of oral health, $55 \%$ worried about their dental appearance, $11 \%$ had difficulty in chewing, 33\% had sensitivity and 23\% experienced dental pain. Surveying this diverse group of patients and dentists suggests that perhaps the opinion of oral health between these two groups are not in accord. As the authors point out it may prove to be a useful predictor of service uptake.

Both the profession and politicians agree that dental treatment and provision of dental services must be based on evidence, yet at present this can be at in many cases, empirical. However, obtaining this evidence is not easy and requires more and bigger studies like this one. It also has to closely involve those that provide the majority of dental services, namely, general dental practitioners. Many people would argue that at present GDPs have neither the knowledge nor skills to carry out meaningful research. The model used in this study, brought together experienced and respected academic researchers with GDPs into a network. The results demonstrate that this model does work and can produce extremely valuable work.

I would hope that those holding the purse strings will further fund this research network to make the best use of the data set and follow up the project into a longitudinal study. Additionally, further funds and efforts should be made to establish GDP research networks in other parts of the UK. It could be money well spent.

\section{Martin Fulford} GDP, Somerset 